\title{
Implicações da mamoplastia de aumento via transaxilar na pesquisa de linfonodo sentinela: revisão da literatura
}

\author{
Implications of transaxillary breast augmentation in the sentinel lymph node \\ detection: literature review
}

\author{
Níveo STEFFen ${ }^{1}$ \\ André Alves Valiati \\ Gustavo de Azambuja \\ Pereira Filho ${ }^{2}$ \\ Tiago Falcão Cunha ${ }^{2}$ \\ PEDRO Bins ElY ${ }^{3}$
}

Trabalho realizado no Serviço de Cirurgia Plástica do Complexo Hospitalar Santa Casa de Porto Alegre - Disciplina de Cirurgia

Plástica da Universidade Federal de Ciências da Saúde de Porto Alegre - UFCSPA, Porto Alegre, RS, Brasil.

Artigo submetido no SGP (Sistema de Gestão de Publicações) da

RBCP.

Artigo recebido: 30/10/2010 Artigo aceito: $15 / 2 / 2011$

\begin{abstract}
RESUMO
Introdução: A pesquisa do linfonodo sentinela é o tratamento padrão para o estadiamento da axila em pacientes com câncer de mama precoce, e sua identificação pode evitar um esvaziamento axilar desnecessário. A incidência de câncer de mama vem aumentando a cada ano, concomitantemente, a mamoplastia de aumento transaxilar vem ganhando cada vez mais adeptos. Objetivo: Revisão bibliográfica com vistas a avaliar se a mamoplastia de aumento transaxilar poderia modificar a drenagem linfática e prejudicar a identificação do linfonodo sentinela. Método: Revisão da literatura. Conclusão: A maioria dos estudos tende a excluir a hipótese de que a mamoplastia de aumento transaxilar possa afetar a identificação do linfonodo sentinela no futuro.
\end{abstract}

Descritores: Linfonodos. Mamoplastia. Neoplasias de mama.

\begin{abstract}
Background: The sentinel lymph node biopsy is the gold-standard technique for axillary staging in patients with early breast cancer, and its identification can avoid an unnecessary axillary lymph node dissection. The incidence of breast cancer is increasing each year; concomitantly, cosmetic transaxillary breast augmentation is gaining much more followers. Objective: We conducted a literature review in order to evaluate if transaxillary breast augmentation can modify the lymphatic drainage and impair the identification of sentinel lymph node. Methods: Review of the literature. Conclusion: The majority of the studies tend to exclude the hypothesis that the transaxillary breast augmentation could affect the identification of sentinel lymph node in the future.
\end{abstract}

Keywords: Lymph nodes. Mammaplasty. Breast neoplasms.

1. Cirurgião Plástico; Preceptor do Serviço de Cirurgia Plástica do Complexo Hospitalar Santa Casa de Porto Alegre, Porto Alegre, RS, Brasil.

2. Médico do Serviço de Cirurgia Plástica do Complexo Hospitalar Santa Casa de Porto Alegre, Porto Alegre, RS, Brasil.

3. Doutorado; Chefe do Serviço de Cirurgia Plástica do Complexo Hospitalar Santa Casa de Porto Alegre; Professor Adjunto da Disciplina de Cirurgia Plástica da Universidade Federal de Ciências da Saúde de Porto Alegre (UFCSPA), Porto Alegre, RS, Brasil. 


\section{INTRODUÇÃO}

O conceito de linfonodo sentinela (LS) foi introduzido por Cabanas ${ }^{1}$, em 1977, para pacientes com câncer de pênis. Consiste no primeiro linfonodo de uma base linfonodal a receber drenagem linfática de certa região anatômica. Teoricamente, o LS traduz o estado de toda base linfonodal em que se encontra. Em 1992, Morton et al. ${ }^{2}$ introduziram a mesma ideia para pacientes com melanoma cutâneo e, em 1994, Giuliano et al. ${ }^{3}$ relataram a eficácia da pesquisa do LS para pacientes com neoplasia maligna da mama.

Especificamente no câncer de mama, a histologia dos linfonodos axilares é um dos fatores prognósticos mais importantes, afetando diretamente o tratamento a ser proposto. A pesquisa do LS está indicada nos casos de câncer de mama inicial, uma vez que estas pacientes geralmente apresentam axilas clinicamente negativas. As principais contraindicações ao procedimento são linfonodos palpáveis ("axila positiva"), radioterapia prévia, procedimentos ou traumatismos axilares prévios e gestação $0^{4,5}$. Doença multicêntrica e tamanho tumoral não são mais contraindicações, desde que a paciente não apresente linfonodos axilares palpáveis ${ }^{5}$. A pesquisa do LS é um procedimento simples, realizado de maneira ambulatorial, e que pode evitar um esvaziamento axilar desnecessário. Quando a pesquisa do LS evidencia linfonodo sem metástases, livra a paciente da cirurgia de esvaziamento axilar, procedimento este bastante mórbido, com índices de complicações de até $80 \%$, sendo mais comumente encontrado edema linfático do membro superior, parestesia, diminuição da mobilidade do ombro e dor crônica ${ }^{4-6}$.

A pesquisa do LS pode ser realizada de três maneiras: injeção periaoreolar de azul patente, de tecnécio ou de ambos marcadores associados. A sensibilidade varia com cada uma das técnicas. O emprego apenas de azul patente gera $65 \%$ de sensibilidade; a injeção de tecnécio gera $71 \%$ de sensibilidade; já a associação das técnicas pode gerar até $95 \%$ de sensibilidade ${ }^{7,8}$. A Figura 1 exemplifica a sequência para identificação do LS.
A mamoplastia de aumento via transaxilar foi introduzida por Hoehler, em 1973, sendo modificada por Peterson, em 1979, o qual inseriu o implante em posição retromuscular ${ }^{9}$. Esta técnica vem ganhando cada vez mais adeptos devido ao resultado estético e ao fato da cicatriz alojar-se fora do território mamário. Considerando que o câncer de mama vem apresentando aumento de incidência nos últimos anos, encontraremos cada vez mais pacientes com câncer de mama e que foram submetidas à mamoplastia de aumento por via transaxilar. Uma dúvida bastante pertinente é se estamos ou não alterando a conduta frente a uma eventual neoplasia maligna da mama que venha a acometer nossas pacientes.

O objetivo deste estudo é realizar uma revisão bibliográfica com vistas a avaliar se a mamoplastia de aumento via transaxilar estaria implicada em modificações da drenagem linfática, prejudicando assim a identificação do LS em pacientes que venham a desenvolver neoplasia maligna da mama.

\section{MÉTODO}

Realizada pesquisa no PubMed com os seguintes descritores: "sentinel lymph node" e "transaxillary breast augmentation".

Foram introduzidos no estudo artigos com os seguintes limitadores: língua inglesa e período de janeiro 1996 a outubro de 2010.

\section{RESULTADOS}

O resultado da pesquisa no PubMed evidenciou 8 estudos entre os anos de 2003 e 2008 (Figura 2). Os oito artigos distribuíram-se da seguinte maneira: 3 relatos de casos $^{9-11}$, 2 estudos de coorte ${ }^{12,13}, 2$ discussões a artigos prévios ${ }^{14,15}$ e 1 estudo anatômico ${ }^{16}$. Destacaremos aqui os seis estudos originais.
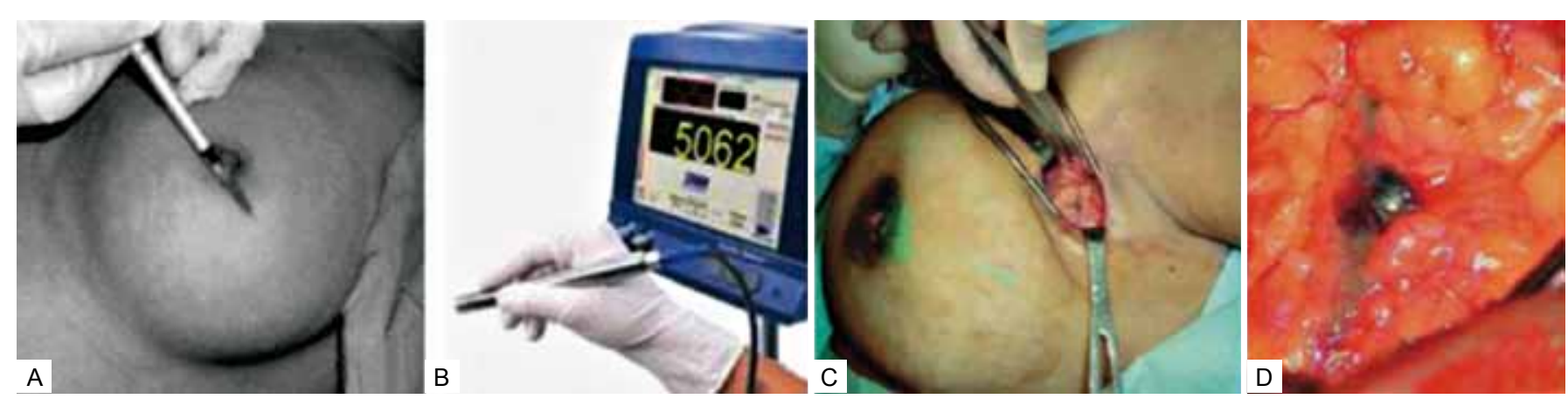

Figura 1 - Sequência para pesquisa do linfonodo sentinela. (A) Injeção de azul patente elou de radioisótopo; (B) Detector de radiação gama; (C) Identificação do linfonodo sentinela; (D) Detalhe do linfonodo sentinela tingido de azul patente. 


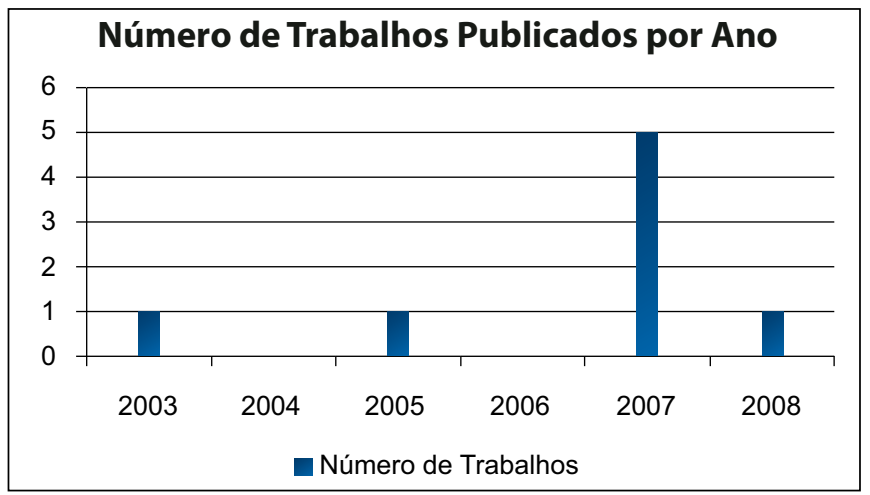

Figura 2-Resultados PubMed para "sentinel lymph node" $e$ "transaxillary breast augmentation", com os limitadores lingua inglesa e periodo de 1996 a 2010 (n=8). Número de trabalhos publicados por ano.

Em 2003, Huang et al. ${ }^{9}$ relataram o caso de uma paciente de 39 anos submetida à mamoplastia de aumento por via transaxilar e que evoluiu com neoplasia maligna da mama alguns meses após a cirurgia estética. A pesquisa de LS foi realizada com sucesso por duas vezes, sendo que a conclusão dos autores foi de que a cirurgia não alterou a pesquisa do LS.

Munhoz et al. ${ }^{10}$ realizaram pesquisa de LS em uma paciente hígida de 28 anos, em avaliação pré-operatória de mamoplastia de aumento por via axilar. A pesquisa de LS foi realizada por meio de linfocintilografia com injeção de tecnécio periaoreolar uma semana antes da cirurgia, bem como com 15 dias e com sete meses de pósoperatório. Os autores concluíram que o mapeamento do LS em pacientes previamente submetidas à mamoplastia de aumento por via axilar é possível.

Em 2007, os mesmos autores publicaram estudo de coorte prospectivo com 26 pacientes hígidas, as quais foram submetidas à linfocintilografia sete dias antes da mamoplastia de aumento por via transaxilar e 10 dias após o procedimento ${ }^{12}$. Os autores concluíram que o mapeamento do LS em pacientes submetidas à mamoplastia transaxilar é possível na maioria dos casos.

Prado et al. ${ }^{16}$ realizaram estudo em 6 cadáveres frescos com o intuito de avaliar se a inserção de implantes por via axilar danificaria os ductos linfáticos. Segundo os autores, devido às forças de tração e de contratração, a introdução de implante de silicone tenderia a danificar os ductos linfáticos, prejudicando a identificação do LS no futuro. Já as próteses salinas, uma vez que são inseridas vazias, não provocariam tais danos, sendo estas mais seguras.

Mottura e Del Castillo ${ }^{11}$ publicaram, em 2007, o relato de dois casos de pacientes submetidas à mamoplastia de aumento por via axilar que evoluíram posteriormente com câncer de mama. Nas duas pacientes, a identificação do LS foi realizada com sucesso, sendo as mesmas tratadas com cirurgia conservadora da mama. Os autores concluíram que a mamoplastia de aumento via transaxilar não prejudica uma futura pesquisa de LS para definir o status da base linfonodal axilar em pacientes que evoluem com câncer de mama.

Em 2008, Sado et al. ${ }^{13}$ publicaram uma coorte prospectiva de 20 pacientes, nas quais foram realizadas linfocintilografias de pré-operatório ( 7 dias antes da cirurgia), pós-operatório precoce (30 dias) e pós-operatório tardio (6 meses). A análise estatística não evidenciou diferença significativa entre as linfocintilografias. Com isso, os autores concluíram que os achados são contrários à hipótese de que a mamoplastia de aumento transaxilar geraria danos à drenagem linfática da axila.

A Tabela 1 resume o delineamento, o país dos autores, o ano de publicação, a revista e a conclusão dos principais artigos aqui descritos.

Tabela 1 - Resumo dos principais estudos descritos nesta publicação.

\begin{tabular}{l|c|c|c|c}
\hline \multicolumn{1}{c|}{ Revista } & Ano & País & Delineamento & Conclusão \\
\hline Aesthetic Surg J & 2003 & USA & Relato de caso (1) & Não afeta a pesquisa do LS \\
\hline Aesth Plast Surg & 2005 & Brasil & Relato de caso (1) & Não afeta a pesquisa do LS \\
\hline Aesth Plast Surg & 2007 & Chile & Estudo anatômico & Afeta a pesquisa do LS \\
\hline Ann Plast Surg & 2007 & Brasil & Estudo de coorte (26) & Não afeta a pesquisa do LS \\
\hline Aesth Plast Surg & 2007 & Argentina & Relato de caso (2) & Não afeta a pesquisa do LS \\
\hline Aesth Plast Surg & 2008 & Brasil & Estudo de coorte (20) & Não afeta a pesquisa do LS \\
\hline
\end{tabular}

$\mathrm{n}=$ número de pacientes; $\mathrm{LS}=$ linfonodo sentinela. 


\section{DISCUSSÃO}

Segundo dados da ISAPS (The International Society of Aesthetic Plastic Surgery), a mamoplastia de aumento foi o segundo procedimento invasivo mais realizado no ano de 2009 , tanto no Brasil quanto no mundo ${ }^{17}$. Não existem dados específicos sobre a via de inserção escolhida em cada uma destas cirurgias, mas certamente o número de adeptos da cirurgia transaxilar vem crescendo ano a ano.

Desde a descoberta de sua efetividade no estadiamento da axila no câncer de mama precoce ${ }^{3}$, a pesquisa do LS se tornou tratamento padrão. Sua importância está no fato de que um LS negativo determina uma base linfonodal livre de doença, evitando uma linfadenectomia axilar desnecessária.

A preocupação sobre a efetividade de uma futura pesquisa de LS em pacientes previamente submetidas à mamoplastia de aumento não é uma novidade. Em 2004, Gray et al. ${ }^{18}$ publicaram estudo retrospectivo com 39 pacientes submetidas previamente a mamoplastia de aumento que evoluíram com câncer de mama. Destas 39 pacientes, 11 realizaram pesquisa de LS, todas com sucesso. No entanto, nenhuma destas 11 pacientes havia sido submetida à técnica transaxilar. No mesmo ano, Jakub et al. ${ }^{19}$ relataram $100 \%$ de sensibilidade na detecção do LS em 49 pacientes submetidas previamente a mamoplastia de aumento. Todavia, os autores não estratificaram as pacientes por via de inserção, e também não deixaram claro quantas pacientes haviam sido submetidas à técnica transaxilar. Em 2009, Rodriguez Fernandez et al. ${ }^{20}$ relataram $100 \%$ de efetividade na deteç̧ão do LS em 70 pacientes submetidas a mamoplastia previamente, sendo 50 mamoplastias de aumento (nenhuma por via axilar) e 20 mamoplastias redutoras.

A maioria dos trabalhos existentes na literatura que associam pesquisa de LS e mamoplastia de aumento, especificamente pela via transaxilar, é fraca do ponto de vista epidemiológico (níveis de evidência 4 e 5). As exceções são os dois trabalhos de coorte publicados no Brasil em 2007 e $2008^{12,13}$.

Em 2007, Munhoz et al. ${ }^{12}$ publicaram estudo de coorte prospectivo com 26 pacientes hígidas, as quais foram submetidas à linfocintilografia sete dias antes da mamoplastia de aumento por via transaxilar e 10 dias após o procedimento. As 26 pacientes foram submetidas à mamoplastia de aumento bilateral, sendo analisadas 52 axilas separadamente. A linfocintilografia de pré-operatório evidenciou o LN em $100 \%$ dos casos. Já o exame de 10 dias de pós-operatório mostrou o mesmo foco do pré-operatório em 24 pacientes. Em dois casos, o exame de pós-operatório apresentou falhas: em uma paciente o LS não foi visualizado bilateralmente; na outra, o LS não foi detectado na axila esquerda. Os autores concluíram que o mapeamento do LS em pacientes submetidas à mamoplastia transaxilar é possível na maioria dos casos. A crítica feita a este estudo foi de que a linfocintilografia de pós-operatório foi realizada muito precocemente, não permitindo a ideal recanalização dos linfáticos.

Uma coorte prospectiva ${ }^{13}$ de 20 pacientes ( 40 axilas analisadas separadamente) foi publicada em 2008. Foram realizadas linfocintilografias de pré-operatório (sete dias antes da cirurgia), pós-operatório precoce (30 dias) e pós-operatório tardio (seis meses). Todas as pacientes foram submetidas à mesma técnica cirúrgica pela mesma equipe, com introdução de implantes de silicone texturizados, redondos, com perfil alto. A análise estatística não evidenciou diferença significativa entre as linfocintilografias de pré e pós-operatório precoce, de pré e pós-operatório tardio, nem de pós-operatório precoce e tardio. Com isso, os autores concluíram que a pesquisa de LS em pacientes submetidas previamente à mamoplastia de aumento transaxilar é possível. Além disso, atribuíram tais resultados à técnica cirúrgica por eles realizada.

Até o momento, não existem estudos na literatura que estratifiquem a pesquisa do LS por plano de dissecção para inserção do implante (subglandular, submuscular e subfascial), tampouco estudos que estratifiquem a pesquisa de LS pelas três principais vias de inserção (periareolar, sulco mamário e transaxilar).

\section{CONCLUSÃo}

A mamoplastia de aumento por via transaxilar é um procedimento que está criando cada vez mais adeptos dentro da Cirurgia Plástica, uma vez que apresenta bons resultados estéticos com um acesso simples, de fácil reprodutibilidade e sem adicionar incisões na mama ${ }^{10-13}$. A atenção com a manipulação excessiva dos tecidos na região axilar deve ser de preocupação do cirurgião plástico, evitando assim danos aos ductos linfáticos ${ }^{13,16}$. Ainda não existem muitos estudos controlados disponíveis na literatura sobre a real implicação desta técnica cirúrgica numa futura pesquisa de LS. No entanto, os dois estudos de coorte disponíveis até o momento ${ }^{12,13}$, juntamente com a maioria dos estudos com menor força estatística publicados ${ }^{9-11}$, favorecem a hipótese de que a mamoplastia de aumento por via axilar não influencia negativamente a identificação do LS.

\section{REFERÊNCIAS}

1. Cabanas RM. An approach for the treatment of penile carcinoma. Cancer. 1977;39(2):456-66.

2. Morton DL, Wen DR, Wong JH, Economou JS, Cagle LA, Storm FK, et al. Technical details of intraoperative lymphatic mapping for early stage melanoma. Arch Surg. 1992;127(4):392-9.

3. Giuliano AE, Kirgan DM, Guenther JM, Morton DL. Lymphatic mapping and sentinel lymphadenectomy for breast cancer. Ann Surg. 1994;220(3):391-8. 
4. Iglehart JD, Kaelin CM. Diseases of the breast. In: Sabiston. Textbook of surgery: the biological basis of modern surgical practice. New York:Elsevier Saunders;2004. p.867-928.

5. Fortunato L, Mascaro A, Amini M, Farina M, Vitelli CE. Sentinel lymph node biopsy in breast cancer. Surg Oncol Clin N Am. 2008;17(3):673-99.

6. Krag D, Weaver D, Ashikaga T, Moffat F, Klimberg VS, Shriver C, et al. The sentinel node in breast cancer: a multicenter validation study. N Engl J Med. 1998;339(14):941-6.

7. Albertini JJ, Lyman GH, Cox C, Yeatman T, Balducci L, Ku N, et al. Lymphatic mapping and sentinel node biopsy in the patient with breast cancer. JAMA. 1996;276(22):1818-22.

8. Urist MM, Soong SJ. Melanoma and cutaneous malignancies. In: Sabiston. Textbook of surgery: the biological basis of modern surgical practice. New York:Elsevier Saunders;2004. p.781-802.

9. Huang GJ, Hardesty RA, Mills D. Sentinel lymph node biopsy in the augmented breast: role of the transaxillary subpectoral approach. Aesthetic Surg J. 2003;23(3):184-7.

10. Munhoz AM, Aldrighi C, Buschpiegel C, Ono C, Montag E, Fells K, et al. The feasibility of sentinel lymph node detection in patients with previous transaxillary implant breast augmentation: preliminary results. Aesthetic Plast Surg. 2005;29(3):163-8.

11. Mottura AA, Del Castillo R. Transaxillary breast augmentation: two breast cancer patients with successful sentinel lymph node diagnosis. Aesthetic Plast Surg. 2007;31(5):544-9.

12. Munhoz AM, Aldrighi C, Ono C, Buchpiguel C, Montag E, Fells K, et al. The influence of subfascial transaxillary breast augmentation in axillary lymphatic drainage patterns and sentinel lymph node detection. Ann Plast Surg. 2007;58(2):141-9.
13. Sado HN, Graf RM, Canan LW, Romano GG, Timi JR, Matias JE, et al. Sentinel lymph node detection and evidence of axillary lymphatic integrity after transaxillary breast augmentation: a prospective study using lymphoscintography. Aesthetic Plast Surg. 2008;32(6):879-88.

14. GrafRM, Canan LW Jr, Romano GG, TolazziAR, Cruz GA. Re: implications of transaxillary breast augmentation: lifetime probability for the development of breast cancer and sentinel node mapping interference. Aesthetic Plast Surg. 2007;31(4):322-4.

15. Munhoz AM, Ferreira MC. Re: implications of transaxillary breast augmentation: lifetime probability for the development of breast cancer and sentinel node mapping interference. Aesthetic Plast Surg. 2007;31(4):320-1.

16. Prado AP, Andrades P, Leniz P. Implications of transaxillary breast augmentation: lifetime probability of breast cancer development and sentinel node mapping interference. Aesthetic Plast Surg. 2007;31(4):317-9.

17. http://www.isaps.org/uploads/news_pdf/Analysis_iSAPS_Survey2009.pdf.

18. Gray RJ, Forstner-Barthell AW, Pockaj BA, Schild SE, Halyard MY. Breast-conserving therapy and sentinel lymph node biopsy are feasible in cancer patients with previous implant breast augmentation. Am J Surg. 2004;188(2):122-5.

19. Jakub JW, Ebert MD, Cantor A, Gardner M, Reintgen DS, Dupont EL, et al. Breast cancer in patients with prior augmentation: presentation, stage, and lymphatic mapping. Plast Reconstr Surg. 2004;114(7):1737-42.

20. Rodriguez Fernandez J, Martella S, Trifirò G, Caliskan M, Chifu C, Brenelli F, et al. Sentinel node biopsy in patients with previous breast aesthetic surgery. Ann Surg Oncol. 2009;16(4):989-92.

\section{Correspondência para:}

André Alves Valiati

Rua Francisco Ferrer, 504 - apto 43 - Bairro Rio Branco - Porto Alegre, RS, Brasil - CEP 90420-140

E-mail: valiatirs@gmail.com 